\title{
Evaluation of NxTAG Respiratory Pathogen Panel and Comparison with xTAG Respiratory Viral Panel Fast v2 and Film Array Respiratory Panel for Detecting Respiratory Pathogens in Nasopharyngeal Aspirates and Swine/Avian-Origin Influenza A Subtypes in Culture Isolates
}

\author{
K. H. Chan, ${ }^{1}$ K. K. W. To, ${ }^{1,2,3}$ P. T. W. Li, ${ }^{1}$ T. L. Wong, ${ }^{1}$ R. Zhang, ${ }^{1}$ K. K. H. Chik, ${ }^{1}$ \\ G. Chan, ${ }^{1}$ C. C. Y. Yip, ${ }^{4}$ H. L. Chen, ${ }^{1,2,3}$ I. F. N. Hung, ${ }^{5}$ J. F. W. Chan, ${ }^{1,2,3}$ and K. Y. Yuen ${ }^{1,2,3,6}$ \\ ${ }^{1}$ Department of Microbiology, The University of Hong Kong, Pokfulam, Hong Kong \\ ${ }^{2}$ State Key Laboratory for Emerging Infectious Diseases, The University of Hong Kong, Pokfulam, Hong Kong \\ ${ }^{3}$ Carol Yu Centre for Infection, The University of Hong Kong, Pokfulam, Hong Kong \\ ${ }^{4}$ Department of Microbiology, Queen Mary Hospital, Pokfulam, Hong Kong \\ ${ }^{5}$ Department of Medicine, The University of Hong Kong, Pokfulam, Hong Kong \\ ${ }^{6}$ The Collaborative Innovation Center for Diagnosis and Treatment of Infectious Diseases, \\ The University of Hong Kong, Pokfulam, Hong Kong
}

Correspondence should be addressed to K. H. Chan; chankh2@hkucc.hku.hk and K. Y. Yuen; kyyuen@hkucc.hku.hk

Received 16 May 2017; Accepted 26 July 2017; Published 29 August 2017

Academic Editor: Subhash C. Verma

Copyright (C) 2017 K. H. Chan et al. This is an open access article distributed under the Creative Commons Attribution License, which permits unrestricted use, distribution, and reproduction in any medium, provided the original work is properly cited.

\begin{abstract}
This study evaluated a new multiplex kit, Luminex NxTAG Respiratory Pathogen Panel, for respiratory pathogens and compared it with xTAG RVP Fast v2 and FilmArray Respiratory Panel using nasopharyngeal aspirate specimens and culture isolates of different swine/avian-origin influenza A subtypes (H2N2, H5N1, H7N9, H5N6, and H9N2). NxTAG RPP gave sensitivity of 95.2\%, specificity of $99.6 \%$, PPV of $93.5 \%$, and NPV of 99.7\%. NxTAG RPP, xTAG RVP, and FilmArray RP had highly concordant performance among each other for the detection of respiratory pathogens. The mean analytic sensitivity (TCID50/ml) of NxTAG RPP, xTAG RVP, and FilmArray RP for detection of swine/avian-origin influenza A subtype isolates was $0.7,41.8$, and 0.8, respectively. All three multiplex assays correctly typed and genotyped the influenza viruses, except for NxTAG RRP that could not distinguish H3N2 from H3N2v. Further investigation should be performed if $\mathrm{H} 3 \mathrm{~N} 2 \mathrm{v}$ is suspected to be the cause of disease. Sensitive and specific laboratory diagnosis of all influenza A viruses subtypes is especially essential in certain epidemic regions, such as Southeast Asia. The results of this study should help clinical laboratory professionals to be aware of the different performances of commercially available molecular multiplex RT-PCR assays that are commonly adopted in many clinical diagnostic laboratories.
\end{abstract}

\section{Introduction}

Respiratory tract infections (RTIs) are caused by various pathogens that are often indistinguishable from one another by clinical diagnosis. RTIs are a major cause of mortality, morbidity, and hospitalization, especially in children aged below five years, and pose significant economic burden [1-6].
Most RTIs are caused by viruses. Common respiratory viruses include influenza virus, adenovirus, parainfluenza virus, respiratory syncytial virus (RSV), human coronaviruses, human metapneumovirus (hMPV), and rhinovirus $[7,8]$.

Rapid and accurate identification of the causative pathogens of RTIs can help guide treatment decisions, possibly reducing the length of hospital stays and associated 
healthcare costs. Moreover, it aids in epidemiologic tracking of local outbreaks or epidemics by implementing proper infection control measures and administering effective antimicrobial therapy. Rapid laboratory identification of RTI pathogens has been associated with up to $50 \%$ reduction in hospital stays, $30 \%$ reduction in the inappropriate use of antibiotics, and $20 \%$ reduction in unnecessary diagnostic tests and procedures [9]. Multiplexed molecular assays that can concurrently detect multiple pathogens from the same sample in a single reaction have been increasingly adopted in clinical microbiology laboratories in the recent years [10]. Several studies have shown that these molecular tests have superior diagnostic capacity and cost-effectiveness of molecular tests when compared to conventional and other diagnostic methods [11, 12].

For influenza viruses, rapid molecular tests based on polymerase chain reaction (PCR) using primers that target the relatively conserved matrix $(\mathrm{M})$ gene are frequently used for achieving rapid and accurate laboratory diagnosis. However, variations in the gene and amino acid sequences in different influenza virus subtypes due to rapid mutations of the viral RNA polymerase may affect the results of these tests. With the emergence of human infections due to the swineorigin influenza A H3N2 variant $(\mathrm{H} 3 \mathrm{~N} 2 \mathrm{v})$ virus in North America in 2010 and more recently the avian-origin influenza A H7N9 and H5N6 in China [13-15], it would be important to ascertain the analytical sensitivities of these rapid tests for the novel influenza viruses $[16,17]$. In a previous study, we have demonstrated that XTAG RVP has lower sensitivity for detection of H7N9 in patient samples and culture isolates $[16,17]$.

NxTAG Respiratory Pathogen Panel (RPP), CE-IVD (Luminex Molecular Diagnostics, Toronto, Canada), is a recent new qualitative multiplex molecular test for the detection of nucleic acids from multiple respiratory viruses and bacteria extracted from respiratory specimens. It detects 22 viral and bacterial targets including influenza A virus, influenza A virus subtype seasonal $\mathrm{H} 1$, influenza A virus subtype seasonal $\mathrm{H} 3$, influenza A virus subtype swine-origin H1N1pdm09, influenza B virus, RSV A and B, parainfluenza viruses types 1 to 4 , adenovirus, hMPV, rhinovirus/enterovirus, human coronavirus- (HCoV-) 229E, HCoV-OC43, HCoV-NL63, HCoV-HKU1, human bocavirus, Chlamydophila pneumoniae, Mycoplasma pneumoniae, and Legionella pneumophila (Table 1). Although there were several studies about the performance of NxTAG RPP using RUO (research use only) assay, the performance of the NxTAG RPP, CV-IVD, for detection of respiratory viruses, particularly the sensitivity and specificity of influenza A subtypes, remains unclear [18-20]. The purpose of this study is to evaluate the NxTAG RPP, CE-IVD, and compare it with Luminex xTAG Respiratory Viral Panel (RVP) Fast v2 (Luminex Molecular Diagnostics, Toronto, Canada) and FilmArray Respiratory Panel (RP) (bioMérieux, Marcy l'Étoile, France), using nasopharyngeal aspirate (NPA) samples obtained from patients with RTIs and culture isolates of different swine and avian-origin influenza A subtypes (H2N2, H3N2v, $\mathrm{H} 5 \mathrm{~N} 1, \mathrm{H} 5 \mathrm{~N} 6, \mathrm{H} 7 \mathrm{~N} 9$, and H9N2).

\section{Materials and Methods}

2.1. Clinical Specimens. The 133 NPA specimens selectively collected from patients with symptoms and signs of RTIs who were managed in Queen Mary Hospital, Hong Kong, and with clinical PCR results were used in this study. These NPA samples were collected into a viral transport medium as described previously [21] and were tested by direct immunofluorescence (DFA) $\left(\mathrm{D}^{3}{ }^{\circledR}\right.$ Ultra $8^{\mathrm{TM}}$ DFA Respiratory Virus Screening and Identification Kit, Diagnostic Hybrids, Inc. (Quidel), USA) for influenza A virus, influenza B virus, parainfluenza viruses types 1 to 4 , respiratory syncytial virus, human metapneumovirus, and adenovirus, and an aliquot of each NPA was sent to Hong Kong Government Virus Laboratory, the Department of Health, for further testing by RT-PCR for detection of influenza A virus, influenza B virus, parainfluenza viruses types 1 to 4 , respiratory syncytial virus, adenovirus, and rhinovirus/enterovirus, as part of the routine clinical diagnostic protocol. Single RT-PCR for detection of human metapneumovirus, human coronavirus- (HCoV-) 229E, HCoV-OC43, HCoV-NL63, HCoV-HKU1, human bocavirus, Chlamydophila pneumoniae, Mycoplasma pneumoniae, and Legionella pneumophila was used as described previously [22-27]. All these PCR results were used as positive reference. The remaining samples were then recruited for NxTAG RVP evaluation. A sufficient quantity of the samples was used for further testing with xTAG RVP and FilmArray RP.

2.2. Influenza Isolates. Two swine-origin and four avianorigin influenza A human isolates, namely, H1N1pdm09 (A/ 415742/09/H1N1), H3N2v (A/Wisconsin/12/2010), H2N2 (A/ Asia/57/3), H5N1 (A/Vietnam/3028/04), H7N9 (A/Anhui/ $1 / 2013)$, and H9N2 (A/HK/1073/99), were used in this study. Additionally, an H5N6 avian isolate [H5N6 (A/Great Egret/H5N6/HK/2016)] was also included. The viruses were cultured for 2 days in Madin-Darby Canine Kidney (MDCK) cells with tosyl sulfonyl phenylalanyl chloromethyl ketone(TPCK-) treated trypsin $(2 \mu \mathrm{g} / \mathrm{ml})$ (Sigma, St. Louis, MO) as previously described [28]. Aliquots of culture supernatant were frozen at $-80^{\circ} \mathrm{C}$ until use. Serial tenfold dilutions of the virus stock with serum-free minimal essential medium (MEM) (Gibco BRL, Grand Island, NY) were tested in NxTAG RPP, xTAG RVP, and FilmArray RP. The viral M gene genome copy numbers per $\mathrm{ml}$ of each virus dilution were determined by real-time, quantitative RT-PCR (q-PCR) as previously described with modification [29, 30]. Briefly, $5 \mu \mathrm{L}$ purified RNA was amplified in a $25 \mu \mathrm{L}$ reaction containing $0.5 \mu \mathrm{L}$ Superscript III Reverse Transcriptase/Platinum Taq DNA polymerase (Invitrogen, Carlsbad, California, USA), $0.05 \mu \mathrm{L}$ ROX reference dye $(25 \mathrm{mM}), 12.5 \mu \mathrm{L}$ of $2 \mathrm{x}$ reaction buffer, $800 \mathrm{nmol} / \mathrm{L}$ forward primer $\left(5^{\prime}\right.$-GACCRATCCTGTCACCTCTGAC-3' ${ }^{\prime}$ ), $800 \mathrm{nmol} / \mathrm{L}$ reverse primer ( $5^{\prime}$-AGGGCATTYTGGACAAAKCGTCTA-3'), and probe $200 \mathrm{nmol} / \mathrm{L}$ (FAM-5' -TGCAGTCCTCGCTCACTGGGCACG-3'-BHQ1). The thermal cycling conditions were 30 minutes at $50^{\circ} \mathrm{C}$ for reverse transcription and then $2 \mathrm{~min}$ at $95^{\circ} \mathrm{C}$ for RT inactivation/initial denaturation, followed by 50 cycles of $15 \mathrm{~s}$ at $95^{\circ} \mathrm{C}$ and $30 \mathrm{~s}$ at $55^{\circ} \mathrm{C}$. All reactions 
TABLE 1: Detectable pathogens and targets.

\begin{tabular}{|c|c|c|c|}
\hline Viruses & NxTAG RPP & xTAG RVP Fast v2 & FilmArray RP \\
\hline Influenza A virus & $\sqrt{ }$ & $\sqrt{ }$ & $\sqrt{ }$ \\
\hline Subtype (H1, H1pdm09, H3) & $\sqrt{ }$ & $(\mathrm{H} 1, \mathrm{H} 3)$ & $\sqrt{ }$ \\
\hline Influenza B virus & $\sqrt{ }$ & $\sqrt{ }$ & $\sqrt{ }$ \\
\hline Respiratory syncytial virus & $(\mathrm{A}, \mathrm{B})$ & $(\mathrm{A}, \mathrm{B})$ & $\sqrt{ }$ \\
\hline Human coronavirus (229E, OC43, NL63, HKU1) & $\sqrt{ }$ & $\sqrt{ }$ & $\sqrt{ }$ \\
\hline Parainfluenza viruses $1-4$ & $\sqrt{ }$ & $\sqrt{ }$ & $\sqrt{ }$ \\
\hline Human metapneumovirus & $\sqrt{ }$ & $\sqrt{ }$ & $\sqrt{ }$ \\
\hline Adenovirus & $\sqrt{ }$ & $\sqrt{ }$ & $\sqrt{ }$ \\
\hline Human bocavirus & $\sqrt{ }$ & $\sqrt{ }$ & \\
\hline Human rhinovirus/Enterovirus & $\sqrt{ }$ & $\sqrt{ }$ & $\sqrt{ }$ \\
\hline Chlamydophila pneumoniae & $\sqrt{ }$ & & $\sqrt{ }$ \\
\hline Legionella pneumophila & $\sqrt{ }$ & & \\
\hline Mycoplasma pneumoniae & $\sqrt{ }$ & & $\sqrt{ }$ \\
\hline Bordetella pertussis & & & $\sqrt{ }$ \\
\hline Total targets & 22 & 18 & 20 \\
\hline
\end{tabular}

were performed using StepOnePlus Real-Time PCR System (Applied Biosystems, Foster City). For quantitative assay, a reference standard plasmid was prepared by cloning the target $\mathrm{M}$ gene amplicon into pCRII-TOPO vector $\left(\mathrm{TOPO}^{\mathrm{TM}}\right.$ TA Cloning ${ }^{\mathrm{TM}}$ Kit, Invitrogen, San Diego, CA) according to the manufacturer's instructions. The copy number of the standard plasmid was determined based on molar concentration of the plasmid ( 1 molar is equivalent to about $6.0221415 \times 10^{23}$ copies numbers). A series of $6 \log 10$ dilutions, equivalent to 1 $\times 10^{1}$ to $1 \times 10^{6}$ copies per reaction, were then prepared from the reference standard plasmid to generate calibration curves and run in parallel with the test samples. The limit of detection of the $\mathrm{M}$ gene $\mathrm{qRT}$-PCR is 10 copies per reaction at $95 \%$ confidence level. The same virus dilutions were inoculated onto MDCK cells to determine the $50 \%$ tissue culture infective dose (TCID50) using the Reed-Muench method as previously described [28].

2.3. Nucleic Acid Extraction. Nucleic acid was detected using easyMAG extraction platform (bioMérieux, Marcy l'Étoile, France) and extracted for NxTAG RPP and xTAG RVP from the specimens as previously described [31]. Total nucleic acid extraction was isolated by using the NucliSENS easyMAG instrument (bioMérieux). Briefly, $10 \mu \mathrm{l}$ of MS2 internal control was first added to $200 \mu \mathrm{l}$ of sample. The mixture was added to $2 \mathrm{ml}$ of lysis buffer and was incubated for 10 minutes at room temperature. The lysed sample was then transferred to the well of a plastic vessel with $100 \mu \mathrm{l}$ of silica. This was followed by automatic magnetic separation. Nucleic acid was recovered in $110 \mu$ l elution buffer. By using a parallel specimen $(300 \mu \mathrm{l})$, nucleic acid extraction, PCR, and detection were performed in FilmArray according to the manufacturer's instructions. The NxTAG RPP incorporates multiplex Reverse Transcriptase-Polymerase Chain Reaction (RT-PCR) with the Luminex proprietary universal tag sorting system on the Luminex platform for detecting respiratory pathogen targets according to the manufacturer's instructions. Briefly, the extracted total nucleic acid is added to preplated Lyophilized Bead Reagents (LBRs) and mixed to resuspend the reaction reagents. The reaction is amplified via RT-PCR and the reaction product undergoes bead hybridization within the sealed reaction well. The hybridized, tagged beads are then sorted and read on the MAGPIX instrument, and the signals are analyzed using the NxTAG Respiratory Pathogen Panel Assay File for SYNCT ${ }^{\mathrm{TM}}$ Software (Luminex, Austin, Texas, USA).

2.4. Statistical Analysis. Diagnostic sensitivity, specificity, PPV, and NPV of the assays against the positive references and agreement between the assays were calculated by VassarStats: Website for Statistical Computation (http:// vassarstats.net).

\section{Results}

3.1. Clinical Samples and Performance of NxTAG RPP. A total of 133 NPA specimens were tested in this study. The specimens were collected from 75 males and 58 females with a mean age of 32.5 years (range: 2 months to 99 years of age). Of 133 specimens, 75 positives were detected by DFA and 151 positives were detected by PCR [influenza $A$ virus = $16(\mathrm{H} 3=12, \mathrm{H} 1 \mathrm{pdm} 09=3, \mathrm{H} 7=1)$, influenza $\mathrm{B}$ virus = 10 , parainfluenza virus $1=10$, parainfluenza virus $2=10$, parainfluenza virus $3=10$, parainfluenza virus $4=11$, RSV = 11 , hMPV $=11$, adenovirus $=4$, rhinovirus/enterovirus $=28$, human coronavirus- (HCoV-) $229 \mathrm{E}=3, \mathrm{HCoV}-\mathrm{OC} 43=6$, HCoV-NL63 = 1, HCoV-HKU1 = 5, human bocavirus = 9, Chlamydophila pneumoniae =1, Mycoplasma pneumoniae = 4, and Legionella pneumophila $=1$ ]. All 133 specimens were evaluated by NxTAG RPP. Overall, 153 pathogens were identified by NxTAG RPP. Ten positives $(H 1 p d m 09 \times 1, R S V A \times 1$, $\mathrm{P} 1 \mathrm{~F} 1 \times 1$, adenovirus $\times 1, \mathrm{EV} /$ rhinovirus $\times 4$, and bocavirus $\times$ 2) were considered as false positives when compared with positive reference. The sensitivity, specificity, positive predictive value, and negative predictive value of NxTAG RPP for 
detection of respiratory viruses were $95.2 \%$ [95\% confidence level (Cl): 90.4-97.7], 99.6\% (95\% Cl: 99.3-99.8), 93.5\% (95\% $\mathrm{Cl}$ : 88.4-96.5), and 99.7\% (95\% Cl: 99.4-99.9), respectively (Table 2).

\subsection{Performance and Concordance of XTAG RVP and FilmAr-} ray $R P$. Of 133 tested samples, 71 had enough quantity to be used for further testing with xTAG RVP and FilmArray RP. The results of these 71 samples tested by the three assays were then compared. Out of these 71 specimens, 66 were positive and 5 were negative by NxTAG RPP. Both the xTAG RVP and FilmArray RP detected respiratory pathogens in 63 (88.7\%) specimens with 96 and 83 pathogens, respectively. The sensitivity, specificity, positive predictive value, and negative predictive value of xTAG RVP and FilmArray RP were 96.9\%, 99.9\%, 99.0\%, and $99.7 \%$ and $85.3 \%, 99.9 \%, 98.8 \%$, and $98.9 \%$, respectively. When NxTAG RPP was compared with xTAG RVP and FilmArray RP, the Kappa value was 0.97 with $95 \%$ CI of $0.95-0.99$ and 0.93 with $95 \%$ CI of $0.89-0.97$, respectively. When xTAG RVP was compared with FilmArray $\mathrm{RP}$, the Kappa value was 0.95 with $95 \%$ CI of $0.91-0.98$. These results suggested that the three assays were highly concordant as a Kappa value higher than 0.80 indicated concordance.

We have previously shown that xTAG RVP has low sensitivity for detecting H7N9 [16, 17]. In this study, one of the patient specimens with confirmed H7N9 influenza A infection was also not detected by xTAG RVP influenza A but was detected by NxTAG RPP and FilmArray RP influenza A. xTAG RVP has a 1000 and 10 times higher limit of detection for H7N9 than NxTAG RPP and FilmArray RP, respectively (Table 3). These findings show that NxTAG RRP has greatly improved the sensitivity for detecting H7N9 over the xTAG RVP. Besides, NxTAG RPP has also improved sensitivity for detecting other respiratory viruses over xTAG RVP (1 adenovirus and 2 bocavirus). These findings corroborate another recent report on the partial comparison between the NxTAG Respiratory Pathogen Panel Assay and the Luminex xTAG Respiratory Panel Fast Assay v2 [32]. NxTAG RPP detected 12 more pathogens than FilmArray RP in our study, with $50 \%$ $(6 / 12)$ being enterovirus/rhinovirus. The other additional positives detected by NxTAG RPP were adenovirus (2), parainfluenza virus $4 \mathrm{~b}(1)$, hMPV (1), H1N1pdm (1), and $M$. pneumoniae (1). Overall, 8 positives, hMPV (1), P2 (1), P4 (3), enterovirus/rhinovirus (2), and $M$. pneumoniae (1), were not detected by NxTAG RPP. All these positives indeed had high Ct value $(\geq 35)$ that may be lower than the limit of detection by NxTAG RPP. Further study should be required if the primers that are suspected do not detect epidemic strains.

Furthermore, the results of 5 samples (4 H1N1pdm 2009 and 1 H7N9) were reported to be "equivocal" by FilmArray RP (Flu A-pan-1 positive, Flu A-pan-2 negative). These 5 specimens were, in fact, true positive as confirmed by other testing methods. These findings indicated that $\mathrm{M}$ gene (Flu A-pan-1) was more sensitive than NS1 gene (Flu A-pan-2) for detection of H1N1pdm 2009.

3.3. Analytical Sensitivity of Influenza A Culture Isolates. When influenza A virus subtype isolates were tested, the mean (range) analytic sensitivities (viral load log10 copies/ml and $\mathrm{TCID}_{50} / \mathrm{ml}$ ) of NxTAG RPP, xTAG RVP, and FilmArray RP were $3.0(1.8-4.2)$ and $0.7(0.0056-3.2) ; 4.3(3.0-6.4)$ and 41.8 (0.56-178); and $3.4(2.7-4.4)$ and $0.8(0.1-1.78)$, respectively (Table 3 ). All avian- and swine-origin influenza A strains were detected by $M$ gene in NxTAG RPP, xTAG RVP, and FilmArray RP. The swine-origin H1N1pdm09 was correctly genotyped by NxTAG RPP and FilmArray RP. However, $\mathrm{H} 3 \mathrm{~N} 2 \mathrm{v}$ and seasonal $\mathrm{H} 3 \mathrm{~N} 2$ could not be differentiated by NxTAG RPP.

\section{Discussion}

In the recent years, the availability of molecular diagnostic tests has revolutionized the diagnosis and surveillance of infectious diseases and treatment of RTIs. Multiplexed molecular assays simultaneously detect multiple pathogens from the same sample in a single reaction vessel, providing a more comprehensive picture of infection. In this study, the clinical performance of the Luminex NxTAG RPP (CV-IVD) in NPA specimens was found to have high sensitivity (95.2\%) and specificity (99.6\%) for detection of respiratory viruses. These findings are similar to those reported in recent evaluation studies using NxTAG RPP (RUO) kit by other groups [18-20]. The performances of the NxTAG RPP, xTAG RVP, and FilmArray RP were also compared. Their results were highly concordant, with the highest concordance reported between NxTAG RRP and xTAG RVP [Kappa value $=0.97$ (95\% Cl: 0.95-0.99)].

According to the manufacturer, $\mathrm{M}$ gene (Flu A-pan-1) and NS1 gene (Flu A-pan-2) are used for influenza A typing by FilmArray RP. The result is only interpreted as positive when both genes are positive. The result will be interpreted as "equivocal" when only Flu A-pan-1 is positive, and the system will suggest the users to repeat testing by the same assay or other assays. In this study, the results showed that an "equivocal" result by FilmArray RP is most likely to be a true positive. The correct interpretation of these results should be considered to avoid delay in diagnosis and treatment particularly in patients with severe influenza A virus infection.

In general, the analytical sensitivities of the NxTAG RPP and FilmArray RP for the swine- and avian-origin influenza viruses were comparable, but xTAG RVP had more than one log lower analytical sensitivity than NxTAG RPP and FilmArray RP (Table 3). NxTAG RPP also had higher analytical sensitivity for detecting H5N6 than the xTAG RVP but the same sensitivity as FilmArray RP and higher analytical sensitivity for detecting $\mathrm{H} 3 \mathrm{~N} 2 \mathrm{v}$ than xTAG RVP and FilmArray RP. All three multiplex assays accurately typed and genotyped the influenza viruses, except for NxTAG RRP that does not distinguish subtyped H3N2 from H3N2v. This finding was further confirmed by testing another $\mathrm{H} 3 \mathrm{~N} 2 \mathrm{v}$ strain, A/Indiana/08/2011, with $500 \mathrm{TCID}_{50}$. A recent study also showed that NxTAG RPP (RUO) only correctly genotyped $95.5 \%$ of influenza A virus H1N1pdm09 strains [19].

Rapid multiplex RT-PCR assays for respiratory pathogens detection are important for establishing diagnosis to facilitate the implementation of appropriate treatment and infection control measures. Sensitive and specific laboratory diagnosis 


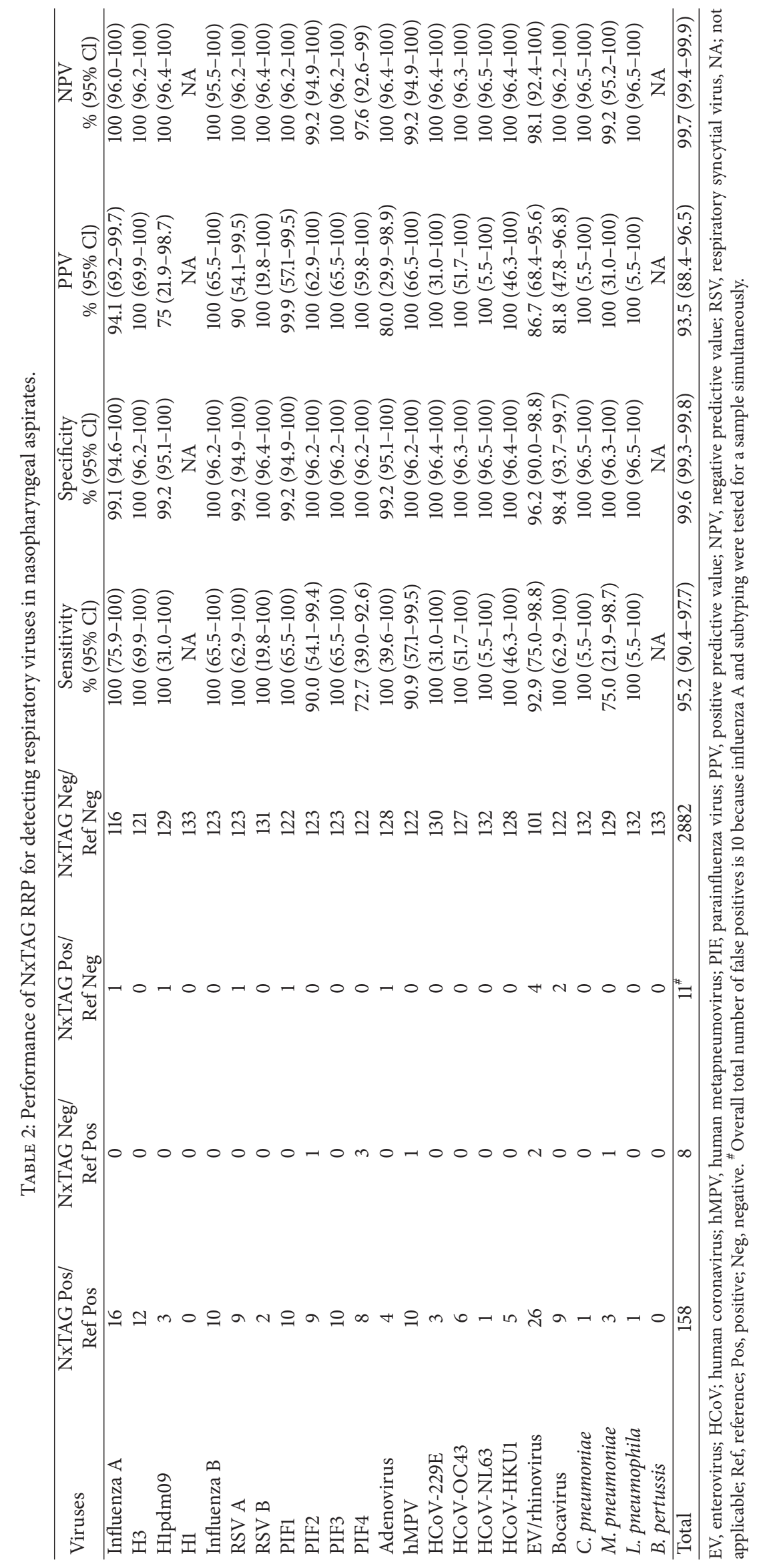




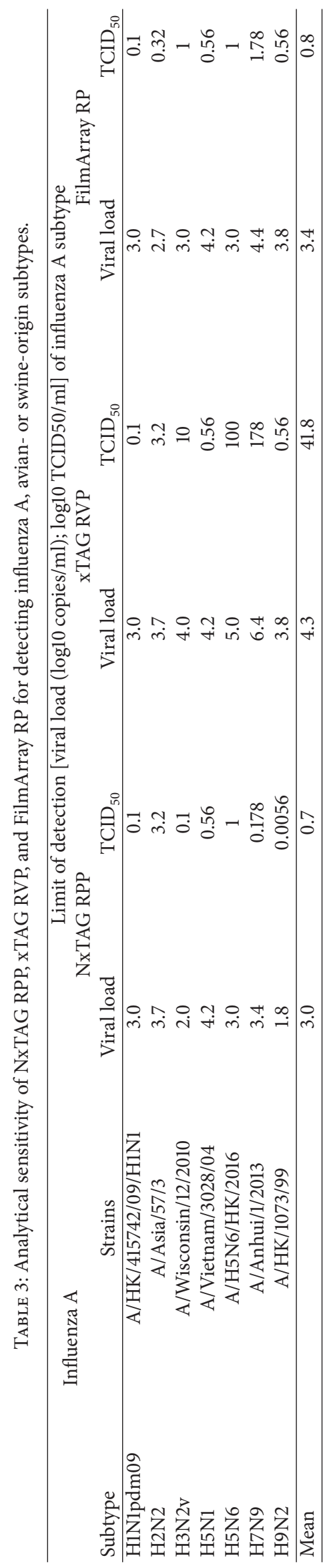


of all influenza A virus subtypes is especially essential in certain epidemic regions, such as Southeast Asia. The correct interpretation of these results is also important and would avoid delay in patient management and infection control, especially in patients with severe seasonal influenza A or avian/swine-origin influenza A virus subtype infection. Clinical microbiologists, infectious disease specialists, and laboratory managers should be aware of and understand the different clinical performances of these commercially available molecular multiplex RT-PCR assays that are commonly adopted in many clinical microbiology laboratories. The sensitivity and specificity of these molecular multiplex assays must be predetermined and be able to confidently detect most of the important avian or swine influenza A subtype infection including $\mathrm{H} 5 \mathrm{~N} 1$ and $\mathrm{H} 7 \mathrm{~N} 9$ and this capability is particularly important for use in Southeast Asia.

A limitation of this study is that some target numbers are small. This is due to low prevalent disease and may require a longer period in order to collect a higher number of positives. High cost for these multiplex assays is another limitation for us to study more samples.

\section{Conclusion}

In this study, the results showed that NxTAG RPP, xTAG RVP, and FilmArray RP assays had high sensitivity and specificity for diagnosis of respiratory diseases. They are suitable for use in clinical diagnostic laboratories for detection of respiratory pathogens in patients with RTIs. However, awareness should be raised that $\mathrm{H} 3 \mathrm{~N} 2$ could not be distinguished from $\mathrm{H} 3 \mathrm{~N} 2 \mathrm{v}$ by NxTAG RPP. Further investigation should be performed if $\mathrm{H} 3 \mathrm{~N} 2 \mathrm{v}$ is suspected to be the cause of disease.

\section{Ethical Approval}

This study was approved by the Institutional Review Board of the University of Hong Kong/Hospital Authority Hong Kong West Cluster.

\section{Conflicts of Interest}

The authors declare that they have no conflicts of interest.

\section{Acknowledgments}

The authors would like to thank the staff of the Department of Microbiology, Queen Mary Hospital, for the facilitation of this project. This work was partly supported by the donations of Larry Chi-Kin Yung and Hui Hoy and Chow Sin Lan Charity Fund Limited; the authors also obtained funding from the Consultancy Service for Enhancing Laboratory Surveillance of Emerging Infectious Diseases, the Department of Health, Hong Kong Special Administrative Region; the University Development Fund and the Committee for Research and Conference Grant; the University of Hong Kong; the Collaborative Innovation Center for Diagnosis and Treatment of Infectious Diseases, the Ministry of Education of China; and the Commissioned Research on Control of Infectious Diseases (Phase III) of the Health and Medical
Research Fund (HKM-15-M-04) of the Food and Health Bureau of the HKSAR Government.

\section{References}

[1] W. Olszewska, M. Zambon, and P. J. M. Openshaw, "Development of vaccines against common colds," British Medical Bulletin, vol. 62, no. 1, pp. 99-111, 2002.

[2] World Health Organization, Burden of Disease Project, World Health Organization, Geneva, Switzerland, 2005.

[3] B. Ehlken, G. Ihorst, B. Lippert et al., "Economic impact of community-acquired and nosocomial lower respiratory tract infections in young children in Germany," European Journal of Pediatrics, vol. 164, no. 10, pp. 607-615, 2005.

[4] M. M. Massin, J. Montesanti, P. Gérard, and P. Lepage, "Spectrum and frequency of illness presenting to a pediatric emergency department," Acta Clinica Belgica, vol. 61, no. 4, pp. 161$165,2006$.

[5] K. G. Nicholson, T. McNally, M. Silverman, P. Simons, J. D. Stockton, and M. C. Zambon, "Rates of hospitalisation for influenza, respiratory syncytial virus and human metapneumovirus among infants and young children," Vaccine, vol. 24, no. 1, pp. 102-108, 2006.

[6] A. Sauro, F. Barone, G. Blasio, L. Russo, and L. Santillo, "Do influenza and acute respiratory infective diseases weigh heavily on general practitioners' daily practice?" European Journal of General Practice, vol. 12, no. 1, pp. 34-36, 2006.

[7] J. S. Tregoning and J. Schwarze, "Respiratory viral infections in infants: causes, clinical symptoms, virology, and immunology," Clinical Microbiology Reviews, vol. 23, no. 1, pp. 74-98, 2010.

[8] S. S. Chiu, P.-L. Ho, M. J. S. Peiris, K. H. Chan, and E. L. Y. Chan, "Population-based hospitalization incidence of respiratory viruses in community-acquired pneumonia in children younger than 5 years of age," Influenza and other Respiratory Viruses, vol. 8, no. 6, pp. 626-627, 2014.

[9] K. J. Henrickson, "Cost-effective use of rapid diagnostic techniques in the treatment and prevention of viral respiratory infections," Pediatric Annals, vol. 34, no. 1, pp. 24-31, 2005.

[10] E. B. Popowitch, S. S. O’Neill, and M. B. Miller, "Comparison of the biofire filmarray RP, Genmark eSensor RVP, Luminex xTAG RVPvl, and Luminex xTAG RVP fast multiplex assays for detection of respiratory viruses," Journal of Clinical Microbiology, vol. 51, no. 5, pp. 1528-1533, 2013.

[11] J. B. Mahony, "Detection of respiratory viruses by molecular methods," Clinical Microbiology Reviews, vol. 21, no. 4, pp. 716747, 2008.

[12] J. B. Mahony, A. Petrich, and M. Smieja, "Molecular diagnosis of respiratory virus infections," Critical Reviews in Clinical Laboratory Sciences, vol. 48, no. 5-6, pp. 217-249, 2011.

[13] Centers for Disease Control and Prevention, Swine-Origin Influenza A (H3N2) Virus Infection in Two Children - Indiana and Pennsylvania, July-August MMWR, 2011.

[14] Y. Chen, W. Liang, S. Yang et al., "Human infections with the emerging avian influenza A H7N9 virus from wet market poultry: clinical analysis and characterisation of viral genome," Lancet, vol. 381, pp. 1916-1925, 2013.

[15] Z.-F. Yang, C. K. P. Mok, J. S. M. Peiris, and N.-S. Zhong, "Human infection with a novel avian influenza A(H5N6) virus," New England Journal of Medicine, vol. 373, no. 5, pp. 487-489, 2015. 
[16] K.-H. Chan, K. K. W. To, J. F. W. Chan, C. P. Y. Li, H. Chen, and K.-Y. Yuen, "Analytical sensitivity of seven point-of-care influenza virus detection tests and two molecular tests for detection of avian origin $\mathrm{H} 7 \mathrm{~N} 9$ and swine origin $\mathrm{H} 3 \mathrm{~N} 2$ variant influenza a viruses," Journal of Clinical Microbiology, vol. 51, no. 9, pp. 3160-3161, 2013.

[17] K.-H. Chan, K. K. W. To, J. F. W. Chan et al., "Assessment of antigen and molecular tests with serial specimens from a patient with influenza A(H7N9) infection," Journal of Clinical Microbiology, vol. 52, no. 6, pp. 2272-2274, 2014.

[18] J. H. K. Chen, H.-Y. Lam, C. C. Y. Yip et al., "Clinical evaluation of the new high-throughput luminex nxtag respiratory pathogen panel assay for multiplex respiratory pathogen detection," Journal of Clinical Microbiology, vol. 54, no. 7, pp. 18201825, 2016.

[19] Y.-W. Tang, S. Gonsalves, J. Y. Sun et al., "Clinical evaluation of the luminex nxtag respiratory pathogen panel," Journal of Clinical Microbiology, vol. 54, no. 7, pp. 1912-1914, 2016.

[20] C. Beckmann and H. H. Hirsch, "Comparing Luminex NxTAGRespiratory Pathogen Panel and RespiFinder-22 for multiplex detection of respiratory pathogens," Journal of Medical Virology, vol. 88, no. 8, pp. 1319-1324, 2016.

[21] I. Hung, V. Cheng, A. Wu et al., "Viral Loads in Clinical Specimens and SARS Manifestations," Emerging Infectious Diseases, vol. 10, no. 9, pp. 1550-1557, 2004.

[22] M. Welti, K. Jaton, M. Altwegg, R. Sahli, A. Wenger, and J. Bille, "Development of a multiplex real-time quantitative PCR assay to detect Chlamydia pneumoniae, Legionella pneumophila and Mycoplasma pneumoniae in respiratory tract secretions," Diagnostic Microbiology and Infectious Disease, vol. 45, no. 2, pp. 85-95, 2003.

[23] L. J. R. Van Elden, A. M. Van Loon, F. Van Alphen et al., "Frequent Detection of Human Coronaviruses in Clinical Specimens from Patients with Respiratory Tract Infection by Use of a Novel Real-Time Reverse-Transciptase Polymerase Chain Reaction," Journal of Infectious Diseases, vol. 189, no. 4, pp. 652657,2004

[24] P. C. Y. Woo, S. K. P. Lau, C.-M. Chu et al., "Characterization and complete genome sequence of a novel coronavirus, coronavirus HKU1, from patients with pneumonia," Journal of Virology, vol. 79, no. 2, pp. 884-895, 2005.

[25] S. S. Chiu, K. H. Chan, K. W. Chu et al., "Human coronavirus NL63 infection and other coronavirus infections in children hospitalized with acute respiratory disease in Hong Kong, China," Clinical Infectious Diseases, vol. 40, no. 12, pp. 1721-1729, 2005.

[26] J. Kuypers, N. Wright, L. Corey, and R. Morrow, "Detection and quantification of human metapneumovirus in pediatric specimens by real-time RT-PCR," Journal of Clinical Virology, vol. 33, no. 4, pp. 299-305, 2005.

[27] S. K. P. Lau, C. C. Y. Yip, T.-L. Que et al., "Clinical and molecular epidemiology of human bocavirus in respiratory and fecal samples from children in Hong Kong," Journal of Infectious Diseases, vol. 196, no. 7, pp. 986-993, 2007.

[28] K. H. Chan, S. Y. Lam, P. Puthavathana et al., "Comparative analytical sensitivities of six rapid influenza A antigen detection test kits for detection of influenza A subtypes H1N1, H3N2 and H5N1," Journal of Clinical Virology, vol. 38, no. 2, pp. 169-171, 2007.

[29] I. F. Hung, K. K. To, J. F. Chan et al., "Efficacy of ClarithromycinNaproxen-Oseltamivir Combination in the Treatment of
Patients Hospitalized for Influenza A(H3N2) Infection," Chest, vol. 151, no. 5, pp. 1069-1080, 2017.

[30] Centers for Disease Control and Prevention, CDC protocol of realtime RTPCR for influenza A (H1N1), Revision 2. Centers for Disease Control and Prevention, Atlanta, GA, 2009.

[31] K. H. Chan, W. C. Yam, C. M. Pang et al., "Comparison of the NucliSens easyMAG and Qiagen BioRobot 9604 nucleic acid extraction systems for detection of RNA and DNA respiratory viruses in nasopharyngeal aspirate samples," Journal of Clinical Microbiology, vol. 46, no. 7, pp. 2195-2199, 2008.

[32] S. Esposito, A. Scala, S. Bianchini et al., "Partial comparison of the NxTAG Respiratory Pathogen Panel Assay with the Luminex xTAG Respiratory Panel Fast Assay V2 and singleplex real-time polymerase chain reaction for detection of respiratory pathogens," Diagnostic Microbiology and Infectious Disease, vol. 86, no. 1, pp. 53-57, 2016. 

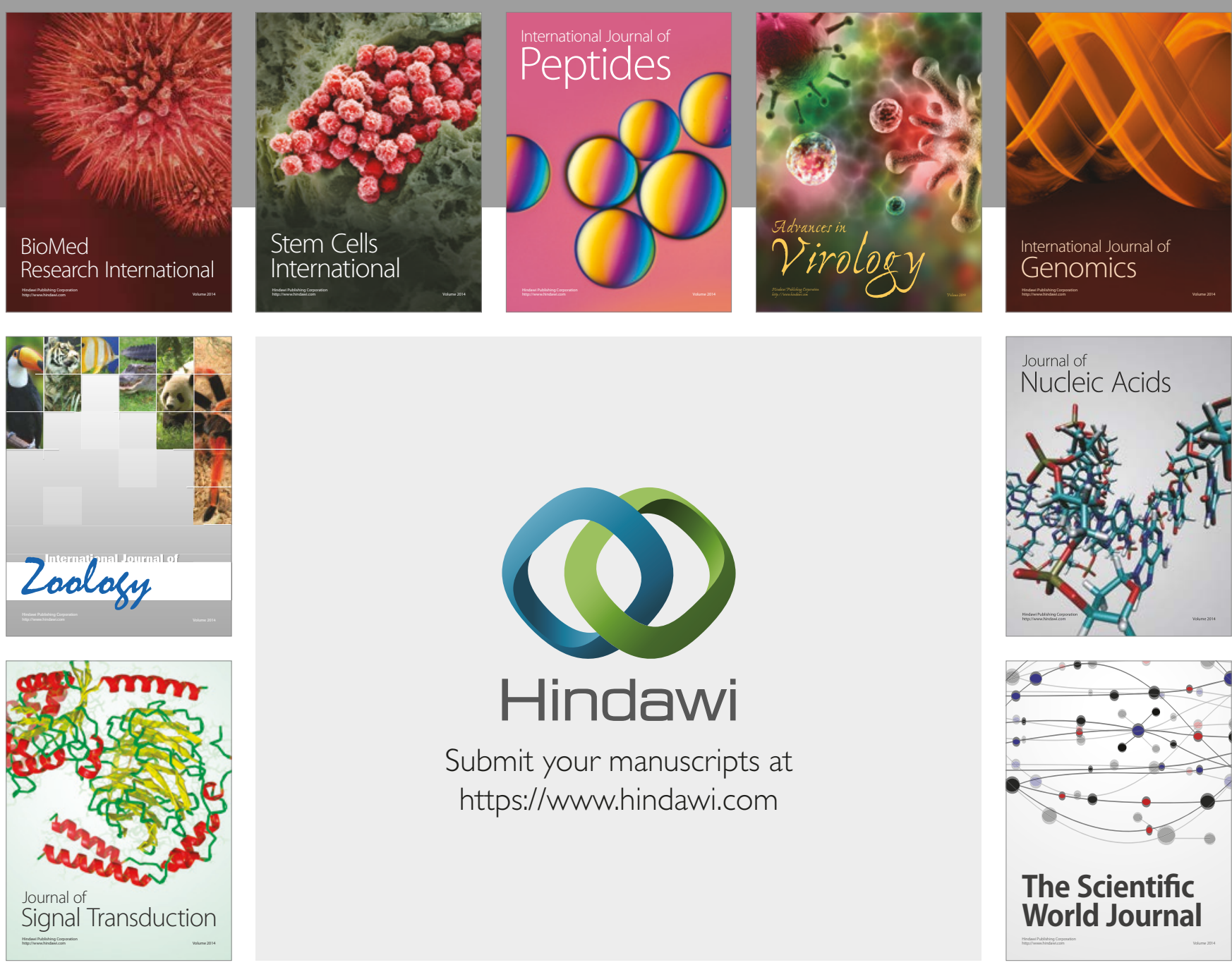

Submit your manuscripts at

https://www.hindawi.com
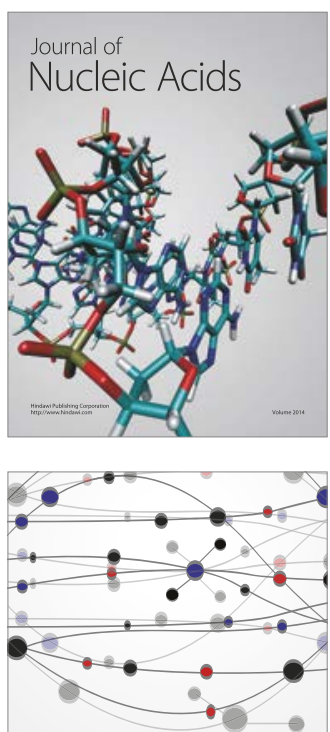

The Scientific World Journal

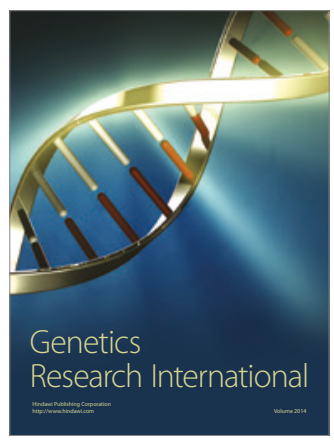

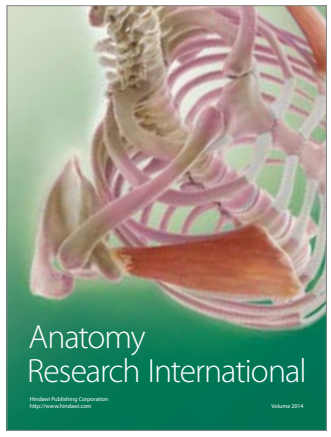

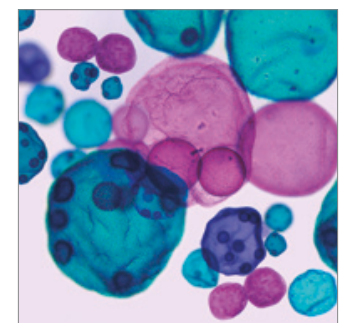

International Journal of Microbiology
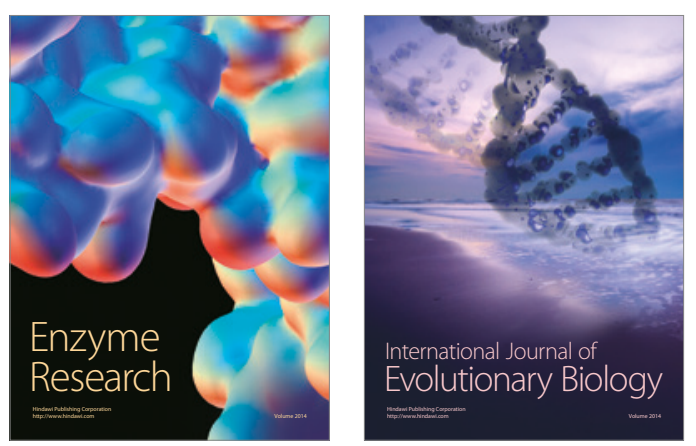
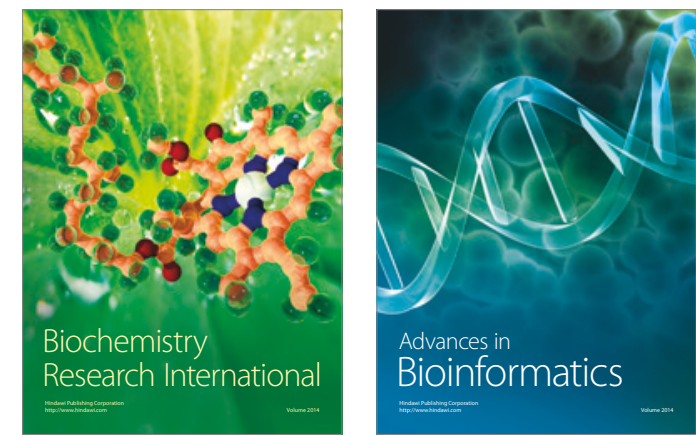

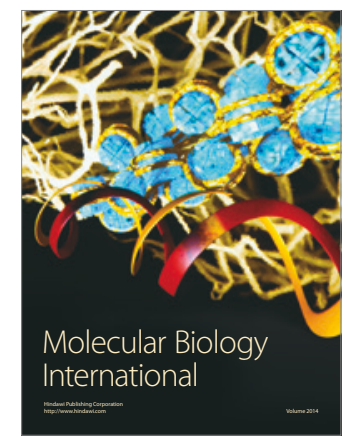

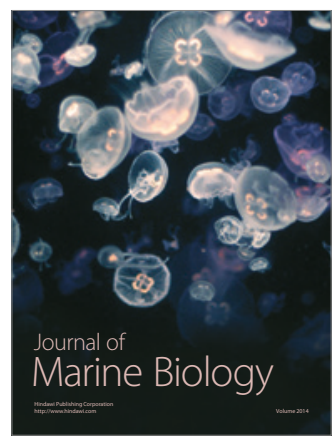

